\title{
Outcomes after corrective surgery for congenital dextro-transposition of the arteries using the arterial switch technique: a scoping systematic review
}

Frederick Morfaw ${ }^{1,2,3^{\wedge}}$, Alvin Leenus ${ }^{1}$, Lawrence Mbuagbaw ${ }^{1,4,5^{*}}$, Laura N. Anderson ${ }^{1}$, Rejane Dillenburg ${ }^{6}$ and Lehana Thabane ${ }^{1,4,6}$

\begin{abstract}
Background: Dextro-transposition of the great arteries (D-TGA) is the most frequent cyanotic congenital heart pathology in neonates. Surgical correction of this condition is possible using the arterial switch operation (ASO) which was first performed by Jatene in 1975.
\end{abstract}

Objectives: The aim of this study was to summarise the evidence on short- (less than 1 year), medium- (1-20 years), and long-term (more than 20 years) outcomes of children with D-TGA treated with the ASO. The primary outcome was survival. Secondary outcomes were freedom from cardiac reoperations, occurrence of aortic insufficiency, pulmonary stenosis, coronary artery anomalies, neuropsychological development problems and quality of life.

Methods: We searched MEDLINE, EMBASE, CINAHL, LILACS, and reference lists of included articles for studies reporting outcomes after ASO for D-TGA. Screening, data extraction and risk of bias assessment were done independently by two reviewers. We pooled data using a random-effects meta-analysis of proportions and, where not possible, outcomes were synthesized narratively. We used the Grading of Recommendations Assessment, Development and Evaluation (GRADE) system to assess the certainty of the evidence for each outcome.

(Continued on next page)

\footnotetext{
* Correspondence: mbuagblc@mcmaster.ca

Frederick Morfaw is deceased.

${ }^{1}$ Department of Health Research Methods, Evidence and Impact, McMaster University, Hamilton, ON, Canada

${ }^{4}$ Biostatistics Unit/FSORC, St Joseph Healthcare-Hamilton, 50 Charlton

Avenue East, 3rd Floor Martha Wing, Room H321, Hamilton, ON L8N 4A6,

Canada

Full list of author information is available at the end of the article
}

(c) The Author(s). 2020 Open Access This article is licensed under a Creative Commons Attribution 4.0 International License, which permits use, sharing, adaptation, distribution and reproduction in any medium or format, as long as you give appropriate credit to the original author(s) and the source, provide a link to the Creative Commons licence, and indicate if changes were made. The images or other third party material in this article are included in the article's Creative Commons licence, unless indicated otherwise in a credit line to the material. If material is not included in the article's Creative Commons licence and your intended use is not permitted by statutory regulation or exceeds the permitted use, you will need to obtain permission directly from the copyright holder. To view a copy of this licence, visit http://creativecommons.org/licenses/by/4.0/ The Creative Commons Public Domain Dedication waiver (http://creativecommons.org/publicdomain/zero/1.0/) applies to the data made available in this article, unless otherwise stated in a credit line to the data. 
(Continued from previous page)

Main results: Following ASO for TGA, short-term survival was 92.0\% (95\% Cl 91.0-93.0\%; $P^{2}=85.8 \%$, 151 studies, 30,186 participants; moderate certainty evidence). Medium-term survival was $90.0 \%$ (95\% Cl 89.0-91.0\%; $P^{2}=84.3 \%, 133$ studies; 23,686 participants, moderate certainty evidence), while long-term survival was $87.0 \%\left(95 \% \mathrm{Cl} 80.0-92.0 \% ; P^{2}=\right.$ 84.5\%, 4 studies, 933 participants, very low certainty evidence). Evaluation of the different secondary outcomes also showed satisfactory results in the short, medium and long term. Subgroup analysis suggests slightly higher survival following ASO for TGA in the second surgical era (1998 to 2018) than in the first surgical era (1975 to 1997$)$ in the short and medium term [93.0\% (95\% Cl 92.0-94.0) vs 90.0\% (95\% Cl 89.0-92.0) and 93.0\% (95\% Cl 91.0-94.0) vs 88.0\% (87.090.0\%) respectively] but not in the long term [81.0\% (95\% Cl 76.0-86.0\%) vs 89.0\% (80.0-95.0\%)].

Conclusions: Pooled data from many sources suggests that the ASO for D-TGA leads to high rates of survival in the short, medium, and long term.

Keywords: Transposition of great arteries, Jatene procedure, Arterial switch operation, Survival, Outcomes, Systematic review registration: PROSPERO CRD42014007590

\section{Background}

\section{Description of the condition}

Dextro-transposition of the great arteries (D-TGA) also known as congenital complete transposition of the great arteries, or simply transposition of the great arteries (TGA) refers to a clinical condition in which the two main arteries leaving the heart (the pulmonary artery and the aorta) are transposed, with the aorta arising from the right ventricle, and the pulmonary artery originating from the left ventricle (ventriculoarterial discordance) [1]. It accounts for $3.0-10.0 \%$ of all congenital heart diseases in children [2]. The reported prevalence varies from 5.0 in 10,000 deliveries in the United States [3] to 1.6 per 10,000 children presenting to the hospital in Nigeria [4]. It is distinct from the levo-transposition of the great arteries (L-TGA) or congenitally corrected TGA which combines both an atrioventricular discordance and a ventriculoarterial discordance, with circulation continuing in the appropriate direction but via the "wrong" ventricles [5].

Unlike L-TGA whose patients may present for the first time in adulthood [6], D-TGA is the most frequent cyanotic congenital heart pathology in neonates and may be rapidly fatal at birth [7]. This is because, in the presence of D-TGA, the systemic and pulmonary circulations function in parallel rather than in series [8]. Consequently, deoxygenated blood from the right ventricle is pumped into the general systemic circulation through the aorta and returns back to the right ventricle; meanwhile, oxygenated blood from the left ventricle is pumped towards the lungs through the pulmonary artery and back to the left ventricle of the heart. Without some connection between the two systems, blood flow is ineffective and the condition will be fatal shortly after birth [8].

Even though physiological connections usually exist between the systemic and pulmonary systems in the fetus (atrial septum, ventricular septum, ductus arteriosus), these usually close up as the fetus approaches maturity. The persistence of these physiological connections ensuring the mixing of blood in the two systems is essential for survival, otherwise, these children become severely cyanosed and are at risk of death [1].

\section{The intervention}

Surgical correction of this condition thereby ensuring the survival of these infants is possible, and the technique has evolved over time. The predominant surgical technique used today is the arterial switch operation (ASO) which was first performed by Jatene in 1975 [9]. The ASO involves repositioning of the great arteries of the heart as well as the coronary arteries, thereby separating the systemic from the pulmonary circulation, and restoring the role of the systemic ventricle to the left ventricle [9].

Conceptually, the ASO better mimics the natural anatomical and physiological circulations of the human circulatory system than the prior atrial switch operations pioneered by Mustard and Senning [10]. However, it is not without complications, which may include left ventricular failure, myocardial ischemia, pulmonary hypertension or supravalvar pulmonary stenosis [9].

\section{Why is it important to do this review?}

With the refinement of the ASO technique over time, there has been a significant improvement in the outcomes of neonates affected by D-TGA. There is however very limited data summarising the long-term outcomes of neonates treated with ASO, and most of these were conducted in single centres. For example, Pretre et al. reported a 10-year survival probability of 93.7\% (95\% confidence interval [CI] 91.4-96.0\%) for neonates undergoing ASO for D-TGA in France [11]. Horer et al. reported 10 - and 20-year survival rates of $92.2 \% \pm 1.3 \%$ and $90.4 \% \pm 1.6 \%$ respectively amongst neonates undergoing ASO for d-TGA in Germany [12]. Khairy et al. reported 25-year survival rates of $96.7 \pm 1.8 \%$ amongst 
neonates undergoing ASO in Boston USA [13]. More recently, Raissadati et al. reported 25-year survival rates of $97.0 \%$ (95\% CI 95.0-100.0\%) among patients undergoing ASO in Finland [14]. While these isolated reports provide a picture of outcomes related to the use of ASO, they are limited by their sample sizes and time frames. Even though ASO has been described as a "great success story", the life-long consequences of this revolutionary surgical technique is purely speculative and needs time [15]. With close to 45 years of use, there is a wealth of information that has accumulated over a reasonable time frame to better inform us of just how successful ASO has been.

In this review, we will provide information on survival and other outcomes after treatment with ASO as outlined in the protocol [16].

\section{Objectives}

The aim of this scoping review was to summarise the evidence on outcomes of children with D-TGA treated with ASO in order to inform clinicians, surgeons, researchers and policymakers on the impact of this technique. Specifically, we wish to determine the short-term (less than 1 year), medium-term (1-20 years) and longterm (more than 20 years) outcomes of children managed by ASO.

\section{Methods}

This review was registered with the International prospective register of systematic reviews (PROSPERO), registration number CRD42014007590 [16]. The methods of this study are published elsewhere [16] but outlined here in brief. The review is reported according to the Preferred Reporting Items for Systematic reviews and Meta-Analyses extension for Scoping Reviews (PRISMA-ScR) (see Appendix 6) [17].

\section{Criteria for considering studies for this review Types of studies}

We included experimental and observational studies which evaluated survival, reoperations, mortality, aortic insufficiency, pulmonary stenosis, residual coronary abnormalities neuropsychological development and quality of life of neonates with D-TGA treated with ASO. For randomised trials, we used only data from the ASO arm. In cases where different technical aspects of the ASO were being compared, we used both arms. We included all studies eligible by the date of the literature search. We excluded commentaries and case reports on patients with D-TGA treated by ASO.

\section{Types of participants}

Our study population included all children born with classic d-TGA diagnosed before or at birth, who underwent ASO. Any studies with only a subset of the relevant participants were included and data extracted only for this subset of participants. We excluded all children undergoing ASO to correct other congenital cardiac anomalies. We equally excluded studies on children with "congenitally corrected" TGA or L-TGA, double outlet right ventricle with sub pulmonary stenosis, visceral heterotaxy and ambiguous atrial situs, right or left atrial isomerism, dextrocardia and situs inversus totalis and superoinferior ventricles.

\section{Type of interventions}

The intervention was ASO with or without the Lecompte manoeuvre (avoidance of the use of a prosthetic conduit in the reconstruction of the pulmonary outflow tract [18]), with or without ventricular septal defect closure, atrial septal defect or patent foramen ovale closure and ductus arteriosus ligation. We excluded studies in which ASO was used as corrective therapy for prior operations, atrial switch operations (Mustard and Senning) [19], Rastelli operation [20], and complex d-TGA repairs (reparation a l'etage ventriculaire or Nikaidoh procedures) [21].

\section{Comparisons}

No comparative data were used.

\section{Outcomes}

Our primary outcome was a survival from the moment of surgery. Secondary outcomes included freedom from cardiac reoperations, occurrence of aortic insufficiency, pulmonary stenosis, coronary artery anomalies, neuropsychological development problems and quality of life measures.

\section{Electronic searches}

Using the OVID search platform, we searched MEDL INE from 1946 to October 2018, and EMBASE from inception (1974) to October 2018. We also searched the Cumulative Index to Nursing and Allied Health Literature (CINAHL) from inception (1981) till October 2018 using the EBSCOhost platform. Finally, we searched the Literature in the Health Sciences in Latin America and the Caribbean (LILACS) database using the Virtual Health Library regional portal from inception (1982) till October 2018. We set no limitations on language or the publication status of the studies. The last search in each of the databases was conducted on the 2nd of October 2018. (See appendix 1 for the MEDLINE search strategy).

\section{Searching other resources}

We hand-searched the reference list of relevant studies and previous reviews as supplemental sources for studies that may have been missed in searches. We searched for grey literature such as unpublished conference proceedings from conference websites such as the Annual Meetings of 
the Spanish Society of Cardiology and the National Turkish Cardiology Conference in order to identify ongoing studies or completed but unpublished studies. Finally, we contacted experts in the field by email for any ongoing studies or relevant but unpublished studies.

\section{Data collection and analysis Study selection}

Two review authors (FM and AL) independently screened the titles and abstracts of all studies identified through the electronic searches in order to identify possible articles for inclusion. Following this screening, the full texts of eligible articles were obtained and assessed based on our inclusion criteria cited above.

\section{Data extraction and management}

We extracted data using a pre-designed and pre-tested data extraction form created using the web-based systematic review software DistillerSR [22]. The data extraction was done in duplicate by two authors (FM and AL) working independently. We extracted data on the study period, the country of conduct of the study, the language, the design, the sample size, the participant characteristics, and the different outcomes of interest as appropriate. In cases where data were reported solely as graphs, we contacted review authors by email for the raw data and, where not possible, we extrapolated directly from the graphs using the online tool WebPlotDigitizer [23]. In cases of incomplete data, missing data or uncertainty, we contacted the authors of the principal studies by email for clarifications. We extracted data on the various outcomes according to three-time frames, short term (less than 1 year), medium term (1-20 years) and long term (more than 20 years).

\section{Assessment of risk of bias in included studies}

We used the risk of bias tool for prevalence studies developed by Hoy et al. [24] to assess the risk of bias in studies included in the review. The tool has 10 items with the first 4 items assessing the external validity of the study (domains of selection and non-response bias). The remaining six items assess internal validity (measurement bias and bias related to analysis). According to the tool, individual items are rated as either at low or high risk of bias. In cases where there was not enough information to make a judgment for a given item, the study was rated as being at a high risk of bias for that item. The overall risk of bias was graded as either low, moderate or high [24]. Risks of bias assessments were done independently by two authors (FM and AL). We resolved any discrepancies in data handling by discussion or by consultation with a third author (LM).

\section{Outcome measures}

Statistical analyses were done using Stata statistical software version 13 [25]. The unit of analysis was the individual. For the primary outcome of survival, we extracted and assessed data on survival or mortality within the short, medium and long term. Secondary outcomes measures included: freedom from reoperation, aortic insufficiency, pulmonary stenosis, coronary artery anomalies, neuropsychological and quality of life outcomes of the infants. Similar to the primary outcome, we extracted and assessed data on these outcomes in the short, medium and long term following ASO as reported in the different studies.

For neuropsychological outcomes, the Bayley Scales for Infant Development version II (BSID-II) is one of the most widely used standardised tests to evaluate developmental retardation [26]. The Mental Development Index (MDI) from the BSID-II is a scale that measures mental abilities, such as memory and communication. The Physical Development Index (PDI) from the BSID-II measures motor abilities, such as body control and dynamic movement [26]. Neuropsychological outcomes we, therefore, extracted in these two domains.

For the quality of life measures, we considered reports which evaluated the quality of life of patients with dTGA operated by the ASO, irrespective of the scale used to evaluate the quality of life. We extracted data on the general perception of health specifically.

\section{Assessment of heterogeneity}

We assessed statistical heterogeneity using visual inspection of the confidence interval of the pooled studies for any overlap, and also the chi ${ }^{2}$ test of homogeneity (statistical significance threshold $P<0.10$ ) and the $I^{2}$ statistic. We rated statistical heterogeneity based on the $I^{2}$ statistic as described in the Cochrane Handbook of Systematic Reviews [27]. According to this guide, an $I^{2}$ between 0.0 and $40.0 \%$ implies heterogeneity might not be important, an $I^{2}$ of between 30.0 and $60.0 \%$ represents moderate heterogeneity, an $I^{2}$ of between 50.0 and $90.0 \%$ represents substantial heterogeneity, while an $I^{2}$ of between 75.0 and $100.0 \%$ represents considerable heterogeneity [27].

\section{Data synthesis}

We pooled the prevalence of reported outcomes across studies using the metaprop command in Stata software [28]. Given the risk of variance instability and undue overestimation of weights in meta-analysis in cases of extreme prevalences (values tending towards $0.0 \%$ or $100.0 \%)$, we pooled our results with prevalence estimates transformed using the Freeman-Tukey double arcsine transformation method [28]. The pooled estimate was then back-transformed to a summary estimate with its associated $95 \%$ confidence interval. In cases of outcomes 
measured on different scales, we standardised the scores to a single scale for meta-analysis. When this was not possible, we converted the values into a $Z$ score which describes outcomes in standard deviation units. The result was a summary $Z$ score which informed us of how many standard deviations the pooled estimate for patients with ASO for TGA differed from the population mean. We used a random-effects model to account for heterogeneity in all the analyses. For outcomes which could not be pooled together, a narrative synthesis of the outcome was conducted.

\section{Subgroup analysis}

We did a subgroup analysis according to the surgical era of operation. This was because we judged that with refinements in diagnostic criteria and the surgical technique, there might be variations in different surgical outcomes depending on the surgical area in which the procedure was conducted. Since the first ASO was performed in 1975 and we ran our study search till 2018, this gave a 43-years span. We, therefore, defined the first surgical area as running from 1975 to 1997 inclusive (first 22 years of ASO). We included in this category all studies in whom most of the patients were judged to have been operated within this timeframe. We defined the second surgical era as the period extending from 1998 until 2018 inclusive and included in this category all studies in whom most of the patients were judged to have been operated within this timeframe.

\section{Sensitivity analysis}

We conducted a sensitivity analysis by pooling the estimates using the untransformed data in order to determine the robustness of our conclusions. For outcomes synthesised by pooling estimates across the $Z$ scores, a sensitivity analysis was not possible as Freeman-Tukey transformations could no longer be done.

\section{Quality of the evidence}

We evaluated the overall quality of the evidence for each of the key outcomes using the Grading of Recommendations Assessment, Development and Evaluation (GRAD E) system for grading evidence described by Guyatt et al. [29]. We used the online GRADEpro Guideline Development Tool (GDT) [30] to construct a "Summary of findings" table (Appendix 4).

\section{Results}

\section{Description of the search outcome}

The electronic databases and other resource search gave us a total of 2115 records after the removal of duplicates. The PRISMA diagram (Fig. 1) summarises the process of screening and selecting studies for inclusion in the review, and the number of studies retained at each stage.
A total of 245 studies were retained for data extraction and included in the data synthesis.

\section{Included studies}

Most of the included studies were retrospective cohort studies with sample sizes ranging from 9 to over 1200 patients, with follow-up times ranging from 1 to 32 years. Appendix 2 (characteristics of included studies) summarises the key features of the included studies.

\section{Excluded studies}

A total of 104 studies were excluded after full-text review. Studies were mostly excluded either because they had the wrong intervention, the wrong study population, they involved the wrong procedure or did not report any outcomes of interest. Appendix 3 (characteristics of excluded studies) summarises the key features of the excluded studies.

\section{Risk of bias in included studies}

We included an assessment of the risk of bias of each of the individual studies in the characteristics of included studies table (Appendix 2). About 30.0\% of the studies were considered to be at high risk of bias for at least one of the outcomes.

\section{Study outcomes}

Table 1 summarises the main study outcomes overall and according to the surgical era (subgroup analysis). A "summary of findings" table for the different outcomes can be found in Appendix 4. Figure 2 summarises the different study outcomes in the short, medium and long term, while Fig. 3 specifically summarises neuropsychological outcomes reported only at medium term.

\section{Survival}

- Short-term survival (0-1 year): The overall shortterm survival rate was 92.0\% (95\% CIs 91.0-93.0\%) with a high level of heterogeneity $\left(I^{2}=85.8 \%, 151\right.$ studies, 30,186 participants, moderate quality evidence). The pooled short-term survival rate in the first era was 90.0\% (95\% CI 89.0-92.0\%) $\left(I^{2}\right.$ $82.6 \%, 62$ studies, 12,875 participants). The pooled short-term survival in the second era was $93.0 \%$ (95\% CI 92.0-94.0\%) ( $I^{2}$ 86.3\%, 89 studies, 17,311 participants).

- Medium-term survival (1-20 years): The overall medium-term survival rate was 90.0\% (95\% CIs 89.0-91.0\%) with a high level of heterogeneity $\left(I^{2}=84.1 \%, 133\right.$ studies, 23,686 participants, moderate quality evidence). The pooled mediumterm survival rate in the first era was $88.0 \%$ (87.0$90.0 \%)$ ( $I^{2} 86.9,70$ studies, 15,403 participants). The 


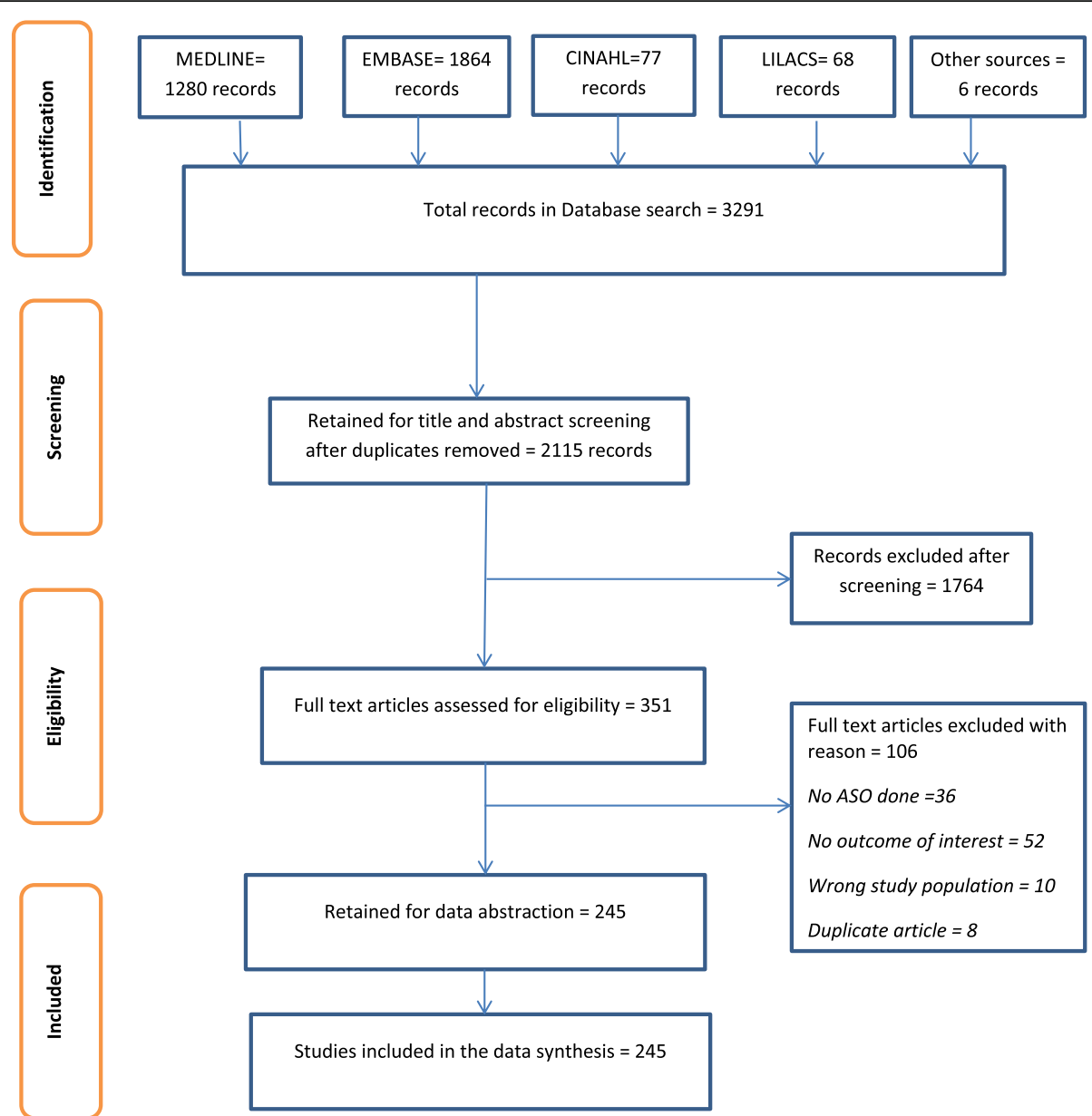

Fig. 1 PRISMA flow diagram

pooled medium-term survival in the second era was 93.0\% (91.0-94.0\%) (I $I^{2} 78.1 \%, 63$ studies, 8283 participants).

- Long-term survival (>20 years): The pooled longterm survival rate was $87.0 \%$ (95\% CIs $80.0-92.0 \%)$ with a high level of heterogeneity $\left(I^{2}=84.5 \%, 4\right.$ studies, 933 participants, very low-quality evidence). The pooled long-term survival rate in the first era was 89.0\% (80.0-95.0) ( $I^{2}$ unavailable, 3 studies, 692 participants). The pooled long-term survival rate in the second era was $81.0 \%$ (76.0-86.0\%) $\left(I^{2}\right.$ not estimable, 1 study, 241 participants).

\section{Freedom from reoperation}

- Short-term freedom from reoperation (0-1 years): The pooled prevalence for short-term freedom from reoperation after ASO for TGA was 93.0\% (95\% CIs 91.0-95.0\%) with a high level of heterogeneity $\left(I^{2}=92.3 \%\right.$, 43 studies, 8391 participants, moderatequality evidence).
- Medium-term freedom from reoperation (1-20 years): The pooled prevalence for medium-term freedom from reoperation after ASO for TGA was 81.0\% (95\% CIs 78.0-84.0\%) with a high level of heterogeneity $\left(I^{2}=95.8 \%, 110\right.$ studies, 21,269 participants, moderate-quality evidence).

- Long-term freedom from reoperation (>20 years): The pooled prevalence for long-term freedom from reoperation after ASO for TGA was 78.0\% (95\% CIs 69.0-86.0\%) with a high level of heterogeneity $\left(I^{2}=95.5 \%, 6\right.$ studies, 2359 participants, very low-quality evidence).

\section{Aortic insufficiency}

- Short-term occurrence of aortic insufficiency (0-1 year): The pooled prevalence for short-term occurrence of aortic insufficiency after ASO for TGA was 4.0\% (95\% CIs 2.0-7.0\%) with a high level of heterogeneity $\left(I^{2}=96.2 \%, 19\right.$ studies, 5354 participants, low-quality evidence). 
Table 1 Summary of main study outcomes

\begin{tabular}{|c|c|c|c|c|c|c|}
\hline \multirow{3}{*}{ Outcome } & \multicolumn{6}{|l|}{ Follow-up } \\
\hline & \multicolumn{2}{|c|}{ Short-term (0-1 year) } & \multicolumn{2}{|c|}{ Medium-term (1-20 years) } & \multicolumn{2}{|c|}{ Long-term (>20 years) } \\
\hline & $\%(95 \% \mathrm{Cl})$ & Studies $\left(I^{2} \%\right)$ & $\%(95 \% \mathrm{Cl})$ & Studies $\left(I^{2} \%\right)$ & $\%(95 \% \mathrm{Cl})$ & Studies $\left(I^{2} \%\right)$ \\
\hline Survival & $92.0(91.0-93.0)$ & $151(85.8)$ & $90.0(89.0-91.0)$ & $133(84.1)$ & $87.0(80.0-92.0)$ & $4(84.5)$ \\
\hline Survival in the first era & $90.0(89.0-92.0)$ & $62(82.6)$ & $88.0(87.0-90.0)$ & $70(86.9)$ & $89.0(80.0-95.0)$ & $3(N / A)$ \\
\hline Survival in the second era & $93.0(92.0-94.0)$ & $89(86.3)$ & $93.0(91.0-94.0$ & $63(78.1)$ & $81.0(76.0-86.0)$ & $1(\mathrm{~N} / \mathrm{A})$ \\
\hline Freedom from reoperation & $93.0(91.0-95.0)$ & $43(92.3)$ & $81.0(78.0-84.0)$ & $110(95.6)$ & $78.0(69.0-86.0)$ & $6(95.5)$ \\
\hline Freedom from reoperation in the first era & $96.0(94.0-97.0)$ & $22(89.4)$ & $83.0(79.0-86.0)$ & $62(95.6)$ & $75.0(63.0-85.0)$ & $4(94.9)$ \\
\hline Freedom from reoperation in the second era & $90.0(85.0-94.0)$ & $21(92.3)$ & $79.0(74.0-84.0)$ & $48(95.9)$ & $85.0(82.0-87.0)$ & $2(N / A)$ \\
\hline Aortic insufficiency & $4.0(2.0-7.0)$ & $19(96.2)$ & $22.0(17.0-26.0)$ & $65(96.9)$ & $29.0(25.0-33.0)$ & $2(\mathrm{~N} / \mathrm{A})$ \\
\hline Aortic insufficiency in the first era & $1.0(0.0-5.0)$ & $11(95.8)$ & $18.0(12.0-25.0)$ & $30(97.6)$ & $41.0(35.0-48.0)$ & $1(\mathrm{~N} / \mathrm{A})$ \\
\hline Aortic insufficiency in the second era & $9.0(2.0-15.0)$ & $8(94.2)$ & $25.0(18.0-32.0)$ & $35(95.9)$ & $19.0(14.0-24.0)$ & $1(\mathrm{~N} / \mathrm{A})$ \\
\hline Pulmonary stenosis & $5.0(2.0-9.0)$ & $9(85.5)$ & $12.0(10.0-15.0)$ & $54(92.8)$ & $82.0(77.0-86.0)$ & $1(\mathrm{~N} / \mathrm{A})$ \\
\hline Pulmonary stenosis in the first era & $8.0(3.0-15.0)$ & $2(N / A)$ & $12.0(9.0-16.0)$ & $28(93.2)$ & - $^{*}$ & $0(N / A)$ \\
\hline Pulmonary stenosis in the second era & $4.0(1.0-8.0)$ & $7(86.5)$ & $12.0(8.0-17.0)$ & $26(92.3)$ & $82.0(77.0-86.0)$ & $1(\mathrm{~N} / \mathrm{A})$ \\
\hline Coronary anomaly & $1.0(0.0-4.0)$ & $5(59.7)$ & $8.0(5.0-11.0)$ & $37(93.3)$ & $23.0(16.0-31.0)$ & $2(N / A)$ \\
\hline Coronary anomaly in the first era & $1.0(0.0-3.0)$ & $1(\mathrm{~N} / \mathrm{A})$ & $4.0(2.0-6.0)$ & $16(87.6)$ & $--^{*}$ & $0(N / A)$ \\
\hline Coronary anomaly in the second era & $1.0(0.0-5.0)$ & $4(53.9)$ & $12.0(7.0-18.0)$ & $21(94.0)$ & $23.0(16.0-31.0)$ & $2(N / A)$ \\
\hline Neuropsychological outcome MDI z score ${ }^{* *}$ & * & - $^{*}$ & $-0.1(-0.8-0.6)$ & $17(0.0)$ & - $^{*}$ & - \\
\hline $\begin{array}{l}\text { Neuropsychological outcome MDI z score** in } \\
\text { the first era }\end{array}$ & - $^{*}$ & 一 $^{*}$ & $-0.0(-0.9-0.8)$ & $10(0.0)$ & - $^{*}$ & $一^{*}$ \\
\hline $\begin{array}{l}\text { Neuropsychological outcome MDI z score** in } \\
\text { the second era }\end{array}$ & 一 $^{*}$ & - $^{*}$ & $-0.3(-1.9-1.2)$ & $7(0.0)$ & - $^{*}$ & - \\
\hline Neuropsychological outcome PDI z score ${ }^{* * *}$ & - $^{*}$ & - $^{*}$ & $-0.3(-1.3-0.7)$ & $11(0.0)$ & - $^{*}$ & - $^{*}$ \\
\hline $\begin{array}{l}\text { Neuropsychological outcome PDI z score }{ }^{* *} \text { in } \\
\text { the first era }\end{array}$ & 一 $^{*}$ & - $^{*}$ & $-0.4(-1.6-0.9)$ & $5(0.0)$ & - $^{*}$ & - \\
\hline $\begin{array}{l}\text { Neuropsychological outcome PDI z score }{ }^{* *} \text { in } \\
\text { the second era }\end{array}$ & - $^{*}$ & - $^{*}$ & $-0.3(-1.9-1.3)$ & $6(0.0)$ & - $^{*}$ & - \\
\hline Quality of life & - $^{*}$ & - $^{*}$ & $\begin{array}{l}\text { Assessed with } 7 \mathrm{~s} \\
\text { studies found no } \\
\text { Health-Related Q } \\
\text { (HRQOL) of ASO } \\
\text { to the general po } \\
\text { studies found tha } \\
\text { had better HRQo } \\
\text { others found the } \\
\text { HRQoL. }\end{array}$ & $\begin{array}{l}\text { studies. Three } \\
\text { difference in } \\
\text { uality of Life } \\
\text { patients relative } \\
\text { opulation; two } \\
\text { at ASO patients } \\
\text { L, while two } \\
\text { y had lower }\end{array}$ & - $^{*}$ & 一 $^{*}$ \\
\hline
\end{tabular}

N/A not applicable (in these cases, the outcome was not computed by the statistical software), MDI Mental Development Index, PDI Physical Development Index, $H R Q O L$ Health-related quality of life, ASO Arterial switch operation.

*No studies reported on this outcome during the specified time frame.

**The $Z$ score compares the number of standard deviations the score of the D-TGA patients differs from that of the general population, with zero being the score of no difference, lower scores being poor compared with the general population, and higher scores being better

- Medium-term occurrence of aortic insufficiency (1-20 years): The pooled prevalence for mediumterm aortic insufficiency after ASO for TGA was $22.0 \%$ (95\% CIs $17.0-26.0 \%)$ with a high level of heterogeneity $\left(I^{2}=96.9 \%, 65\right.$ studies, 10,705 participants, moderate-quality evidence).

- Long-term occurrence of aortic insufficiency $(>20$ years): The pooled prevalence for long-term aortic insufficiency after ASO for TGA was 29.0\% (95\%
CIs 25.0-33.0\%) ( $I^{2}$ unavailable, 2 studies, 461 participants, very low-quality evidence).

\section{Pulmonary stenosis}

- Short-term occurrence of pulmonary stenosis $(0-1$ years): The pooled prevalence for short-term occurrence of pulmonary stenosis after ASO for TGA was 5.0\% (95\% CIs 2.0-9.0\%) with a high level 


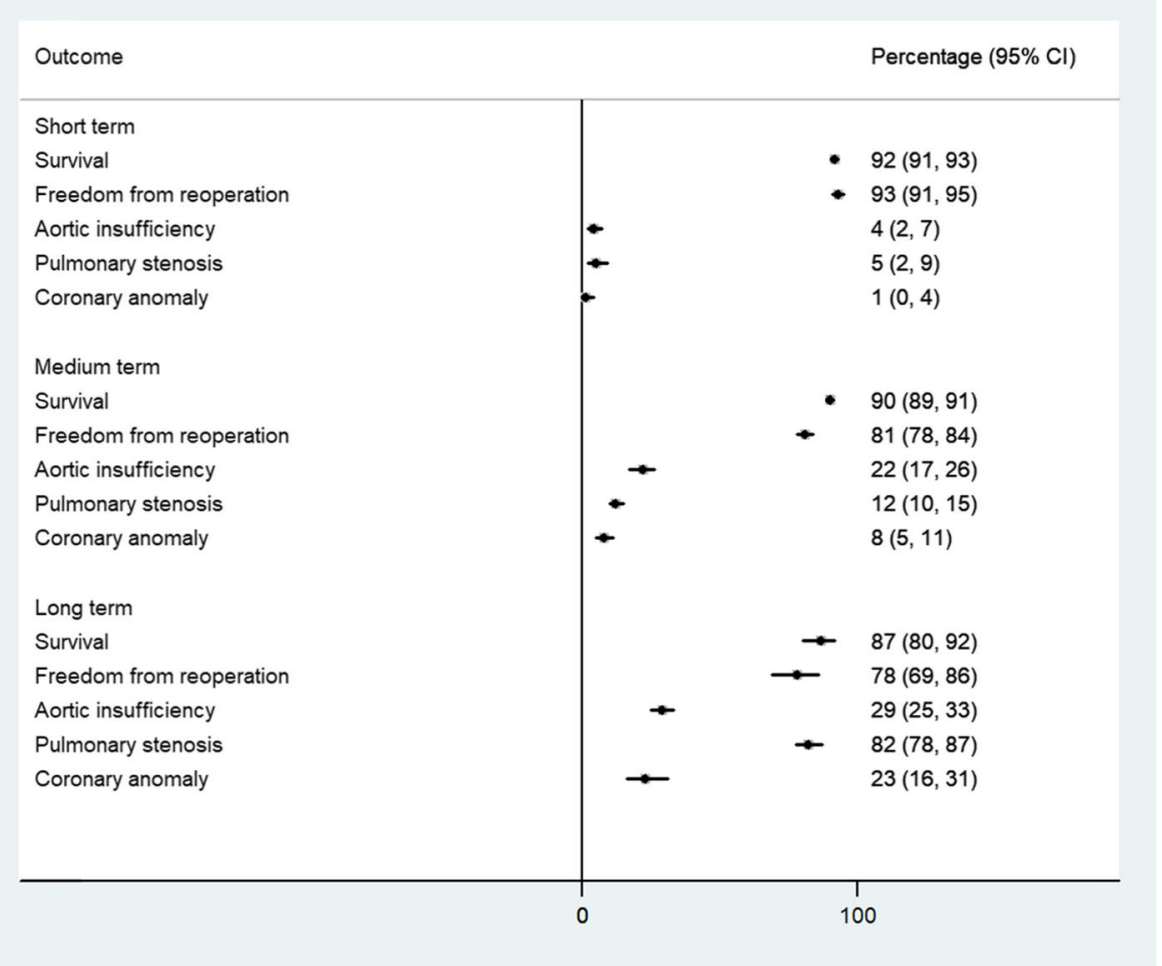

Fig. 2 Summary of different study outcomes in the short term, medium and long term

of heterogeneity $\left(I^{2}=85.5 \%, 9\right.$ studies, 1150 participants, moderate-quality evidence).

- Medium-term occurrence of pulmonary stenosis (1-20 years): The pooled prevalence for mediumterm aortic insufficiency after ASO for TGA was $12.0 \%$ (95\% CIs 10.0-15.0\%) with a high level of heterogeneity $\left(I^{2}=92.8 \%\right.$, 54 studies, 8607 participants, moderate-quality evidence).

- Long-term occurrence of pulmonary stenosis ( $>20$ years): Only one study [31] reported on this outcome. The prevalence of long-term pulmonary stenosis was $82.3 \%$ (95\% CIs 77.5-87.1\%) (1 study, 241 participants, very low certainty evidence). A sensitivity analysis was not conducted for this outcome.

\section{Coronary anomaly}

- Short term occurrence of any coronary anomaly (0-1 years): The pooled prevalence for short term occurrence of any coronary anomaly after ASO for TGA was $1.0 \%(95 \%$ CIs: $0.0-4.0 \%)$ with a moderate level of heterogeneity $\left(\mathrm{I}^{2}=59.7 \%, 5\right.$ studies, 611 participants, low certainty evidence).
- Medium-term occurrence of any coronary anomaly (1-20 years): The pooled prevalence for mediumterm occurrence of any coronary anomaly after ASO for TGA was $8.0 \%$ (95\% CIs 5.0-11.0\%) with a high level of heterogeneity $\left(I^{2}=93.3 \%, 37\right.$ studies, 6542 participants, low-certainty evidence).

- Long-term occurrence of any coronary anomaly ( $>20$ years): The pooled prevalence for long-term occurrence of any coronary anomaly after ASO for TGA was 23.0\% (95\% CIs 16.0-31.0\%) ( $I^{2}$ unavailable, 2 studies, 134 participants).

\section{Neuropsychological outcomes (mental development and physical development) at medium-term (1-20 years)}

- Mental development index: The pooled weighted $Z$ score for the mental development index after ASO for TGA was -0.1 (95\% CIs -0.8-0.6) with a little to no heterogeneity $\left(I^{2}=0.0 \%, 17\right.$ studies, 1419 participants, moderate-certainty evidence).

- Physical development index: The pooled weighted $Z$ score for the physical development index after ASO for TGA was $-0.3(95 \%$ CIs -1.3-0.6) with a little to no heterogeneity $\left(I^{2}=0.0 \%, 17\right.$ studies, 1419 participants, moderate certainty evidence). 


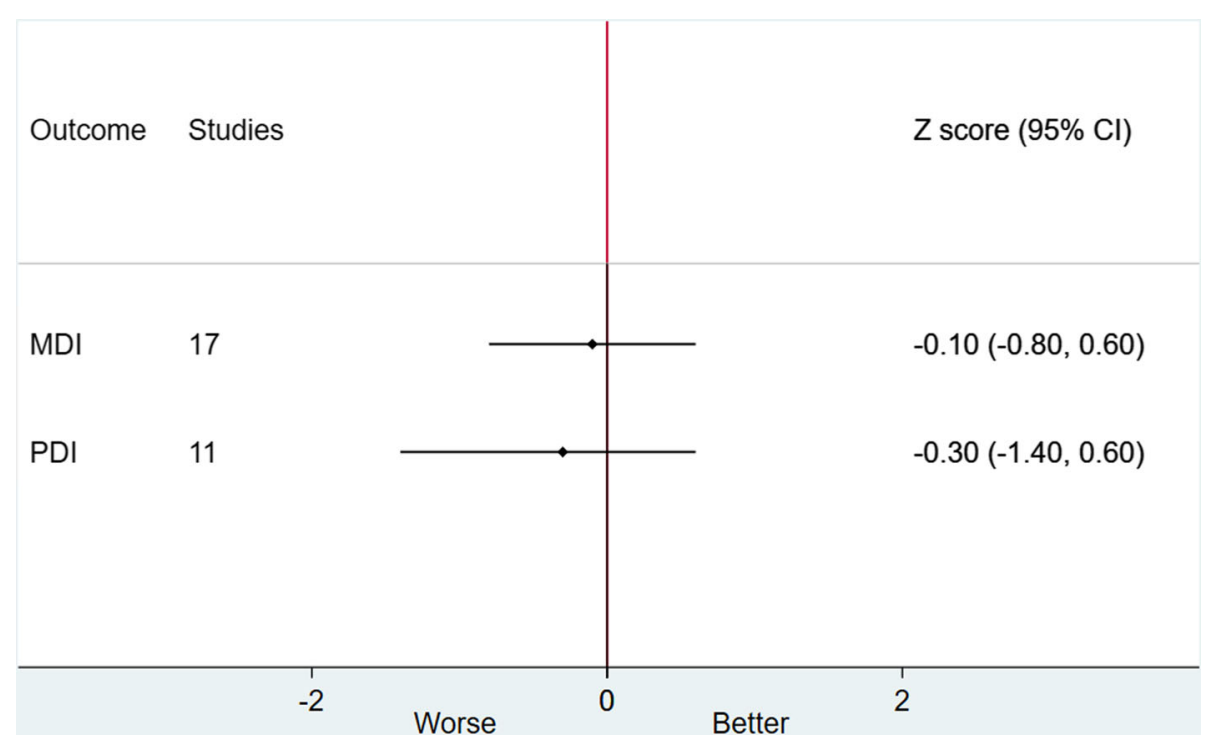

Fig. 3 Summary of neuropsychological outcomes $z$ scores for D-TGA patients at medium term. MDI = Mental Development Index. PDI = Physical Development Index

\section{Quality of life outcomes}

Seven studies evaluated the quality of life of ASO survivors [32-38]. They used different scales and reported on different aspects, hence a meta-analysis of the results was not possible, and the results were narratively summarised (see Table 2). The general tendency was that there was no substantial difference in the quality of life between ASO patients and the comparison groups.

\section{Sensitivity analysis}

A sensitivity analysis using the untransformed prevalence estimates showed similar results for the different outcomes and time frames (see Appendix 5).

\section{Discussion}

\section{Summary of main results}

Following ASO for TGA, short-term survival was 92.0\% (95\% CI 91.0-93.0\%; $I^{2}=85.8 \%, 151$ studies, 30,186 participants; moderate-certainty evidence). Mediumterm survival was $90.0 \%$ (95\% CI 89.0-91.0\%; $I^{2}=84.3 \%$, 133 studies; 23,686 participants, moderate-certainty evidence), while long-term survival was $87.0 \%$ (95\% CI 80.0-92.0\%; $I^{2}=84.5 \%$, 4 studies, 933 participants, very low-certainty evidence). Evaluation of the different secondary outcomes also showed satisfactory results in the short, medium and long-term. Sensitivity analysis showed similar results. Subgroup analysis suggests slightly higher survival following ASO for TGA in the second surgical era (1998 to 2018) than in the first surgical era (1975 to 1997) in the short and medium term [93.0\% (95\% CI 92.0-94.0) vs 90.0\% (95\% CI 89.0-92.0) and $93.0 \%$ (95\% CI $91.0-94.0 \%)$ vs $88.0 \%(87.0-90.0 \%)$ respectively] but not in the long term $[81.0 \%$ (95\% CI $76.0-86.0 \%)$ vs $89.0 \%(80.0-95.0 \%)]$.

\section{Agreement and disagreement with other reviews}

We identified several review articles which had attempted to summarise different outcomes following arterial switch operation [39-46]. However, most of these synthesized the data narratively, and none presented a complete overview of the different outcomes over the different timeframes evaluated in this review.

Our estimates for survival in the short, medium and long term were similar to those from other studies which reported short-term survival rates of about $95.0 \%$ [39, 40], medium-term survival rates of about 88.097.0\% [39-42], and long-term survival rates of about $90.0-96.7 \%$ [39, 42]. Many factors have been reported as being predictors of early survival following ASO for TGA. These include complex anatomy, coronary anomalies, an extended coronary artery bypass time, low APGAR-score, preterm birth, the need for additional procedures, and surgeries performed either too soon or too late $[47,48]$. Our study did not account for these and other predictors of survival. However, our findings suggest improved survival in the second surgical era relative to the first surgical era. It has been hypothesized that improvements in surgical technique, intensive care unit treatment and the better clinical condition of patients presenting in the second era may account for these results [48]. 
Table 2 Summary of quality of life outcomes

Study: Main feature and scale used for quality of life assessment

Fricke et al [32]: Compared the quality of life of 107 ASO patients with that of an age-matched Australian population using Short Form 6-Dimension (SF6D).

Kalfa et al [33]: Evaluated the cognitive and psychological outcomes of 67 ASO adults (18.0-31.0 years) compared to 43 matched controls using the

Short Form 36 (SF-36).

Ruys et al [34]: Reported the quality of life of 18 ASO patients (22.0-25.0 years), and compared them with healthy controls and patients who underwent the Mustard procedure for TGA using the Short Form 36.

Gorler et al [35]: Compared the quality of life of 98 ASO adults survivors against those of atrial repair patients and the general population and reported the $Z$ scores from the Short Form 36.

De Koning et al [36]: Compared the quality of life of 31 ASO child survivors age 8.0 -15.0 years against children of the same age from the general Dutch population using the TNO-AZL child quality of life (TACQOL) questionnaire.

Hovels-Gurich et al [37]: Compared the quality of life of 60 ASO child survivors (mean age 10.5 years) against children of the same age from the general German population using the Inventory for the Assessment of the Quality of Life in Children and Adolescents (IQLC).

Dunbar-Masterson et al [38]: Compared the quality of life of 155 ASO child survivors (median age 8.1 years) against a normative sample of children of the same age from the general American population using the Child Health Questionnaire, Parent Form-50.

\section{Outcome}

No statistically significant difference in the mean SF6D scores between ASO patients and the general Australian population (18-24 age group: [0.769 for ASO patients vs 0.772 for Australian population, $P=0.85$ ]; $25-$ 34 age group [0.795 for ASO patients vs 0.780 for Australian population, $P=0.33])$.

ASO patients had a statistically significant lower physical component summary compared to controls (52.1 \pm 7.5 vs $55.4 \pm 5.9, P=0.01)$. No statistically significant difference in the mental component summary $(47.2 \pm 10.3$ vs $44.5 \pm 11.5)$.

They reported that ASO patients scored significantly better than the normal Dutch population on domains of physical functioning, vitality and role limitations due to emotional problems $(P<0.01)$ [34].

They did not find any statistically significant difference in quality of life between ASO patients and the comparison groups. They however noted a tendency for better results in the categories of "general health" and "role emotional" for the ASO patients [35].

They noted poorer health related quality of life (HRQOL) in motor functioning and positive emotional functioning among ASO children, with no further differences on other TACQOL scales [36].

They did not find any statistically significant difference in total quality of life between ASO children and healthy controls (mean score [standard deviation SD] 1.62 [0.66] vs 1.64 [0.59] $P>0.20$ ) [37].

They found similar Physical Health Summary (mean score [SD] 54.0 [6.1] vs 53.0 [8.8]) and Psychosocial Summary scores (mean score [SD] 49.7 [9.9] vs 51.2 [9]) between ASO children and healthy controls [38].

ASO Arterial switch operation, TGA Transposition of the great arteries, SF6D Short Form 6-Dimension, SF-36 Short Form 36, TACQOL TNO-AZL child quality of life questionnaire, HRQOL Health-related quality of life, IQLC Inventory for the Assessment of the Quality of Life in Children and Adolescents

Staying free from cardiac surgical reoperation after ASO for D-TGA may be as important as surviving over the years. Yet few reviews have attempted to gather the evidence on freedom from cardiac surgical reoperation following ASO for TGA. Based on fewer studies than in our report, Tabitha et al. reported a 10-year freedom from reoperation rate at $82.0 \%$ [42], a finding similar to the $81.0 \%$ (78.0-84.0\%) medium-term freedom from reoperation in our study. There is limited evidence on long-term freedom from reoperation for these patients.

The most frequent complication of ASO for TGA is supravalvular pulmonary stenosis [43]. Our estimates for pulmonary stenosis in the short and medium term are similar to those from other studies which reported short-term pulmonary stenosis rates of $4.0-11.0 \%$ [40] and medium-term pulmonary stenosis rates between 2.0 and $30.0 \%$ [39, 43]. Considering the potential morbidity associated with pulmonary stenosis and the need for reintervention in many cases, an improvement in the ASO technique to help minimize this complication is important.

Reports of the prevalence of aortic insufficiency varied across different reviews. Raja et al. reported a midterm prevalence of aortic insufficiency of about $35.0 \%$ [40], a value much higher than the $<7.0 \%$ prevalence rate of severe aortic insufficiency reported by Vargo et al. in their narrative review [44]. Our point estimate of a $22.0 \%$ prevalence of aortic insufficiency does not concur with either of these values. Our value however falls within the wider estimates for aortic insufficiency varying from $0.0-50.0 \%$ reported by Blume et al. [43]. Blume et al. noted that this variation is mainly related to the different diagnostic methods used [43].

The risk of coronary artery occlusion following ASO which can compromise myocardial perfusion is real [44]. Our estimates for coronary artery occlusion in the medium term are similar to those from other studies which reported coronary artery obstructive lesions to range between 2.0 and $11.0 \%$ in the medium term [40, $42,44]$. Our medium-term estimate of coronary artery anomaly of $8.0 \%$ is however higher than the $2.0-5.0 \%$ prevalence rate reported by Blume et al. earlier on [43]. This difference is probably due to the fact that Blume et al. used a narrative review to describe this outcome and fewer studies than those included in our metaanalysis.

Few reviews have reported on child development outcomes following D-TGA. A narrative review by Kasmi et al. reported scores on the Bayley Scales of Infant Development (BSID) to be 0.5-1.0 standard deviation 
(SD) below the expected mean values, suggestive of mild to moderate delays in cognitive, motor and language functions [46]. These contrast with our findings which do not show any statistically significant difference between mean values on the BSID reported by TGA patients post-ASO and their normal counterparts. The summary estimate provided by Kasmi et al. [46] is based on a narrative synthesis of four studies, all of which were included in our meta-analysis, in addition to 13 other studies. Our findings suggest that these patients could have a normal neuropsychological life, at least in the medium term.

In a systematic review assessing the quality of life of adult congenital heart disease patients, Fteropoulli et al. [49] highlighted that methodological limitations of defining and measuring quality of life in different studies hindered valid conclusions to be made in this domain in general. We agree with this conclusion as the studies included in our review used varied tools and tended to measure different aspects of quality of life, making it hard to provide a general conclusion on the quality of life of these patients.

\section{Strengths of this review}

The strength of this review lies in its breadth, providing a concise summary evidence for different outcomes in children treated with ASO for D-TGA. Our findings also find strength in the number of included studies and participants. We employed a comprehensive literature search of pertinent databases without any language restriction. Screening, data extraction and risk of bias assessments were done independently by two reviewers, minimising biases in the data extraction and the evidence synthesis.

\section{Potential limitations in the review}

Given that this review summarises evidence from mostly observational studies with varying methodologies and varying methods of outcome ascertainment and reporting, the overall risk of bias remains pertinent. Furthermore, our study does not adjust for potential risk factors which may lead to variations in the reported outcomes in the different studies. Moreover, advancements in surgical technique and surgical expertise over time suggest that outcomes for this procedure are bound to vary with time. Our review does not capture a time distinction which may mark an evolutionary advancement in the ASO expertise, beyond which patient outcomes may differ from initial cohorts. This is because even though we evaluated outcomes according to the surgical era, this distinction was at best approximative. We did not have access to individual patient data from all included studies to precisely categorize patients according to surgical era. Follow-up periods most likely overlapped across our boundaries for the surgical era, and as such the results of the subgroup surgical era analyses need to be interpreted with caution.

\section{Overall completeness and applicability of the evidence}

We employed a comprehensive and broad search strategy and are confident that the right participants, intervention (ASO), and outcomes have been explored with this evidence. We included a total of 245 studies, making this the most complete body of evidence on outcomes after ASO for D-TGA to date. Most of the studies included in this review were conducted in high- and middle-income countries, and this may limit the applicability of the findings especially in underdeveloped settings where we hardly found evidence for the conduct of ASO. This may reflect publication bias which was detected (result not shown) in the assessment of certain outcomes, thus limiting the overall completeness of our evidence.

\section{Quality of the evidence}

Overall, our judgment of the quality of evidence for the outcomes in this review is moderate to very low (Appendix 4). This is primarily due to the study design of the majority of the included studies, as well as the inconsistencies of results and poor quality of reporting for certain outcomes.

\section{Implications for clinical practice}

Improvements in the diagnostic techniques for TGA are important if these children are to subsequently benefit from the successes of ASO. There is a need for dissemination of the diagnostic and surgical technique such that it may reach all those affected especially in lowincome countries. There is also a need for the continued advancement of the surgical technique and comprehension of the potential complications.

\section{Implications for policy}

Babies undergoing ASO for D-TGA remain a unique group of individuals who need to be closely monitored throughout their lives. The inconsistency and poor quality of reporting on outcomes of these patients seen in different studies suggest a need for improved health policy on surveillance and documentation of outcomes for these patients.

\section{Implications for research}

Our research identified some evidence of publication bias in reporting certain outcomes of ASO patients. It is important for researchers to publish all results, be they good or bad, if we are to have a true picture of the impact of an intervention. Our study highlights a research gap on the limited amount of research done on ASO for TGA in the long term (beyond 20 years). Despite the fact that this procedure has been around for 40 years and 
above, very few studies have done long term follow-up on different outcomes of these patients beyond 20 years.

\section{Conclusions}

The ASO for TGA leads to high survival rates and few morbidities. More long-term research is encouraged to monitor outcomes throughout the duration of life for children who receive the ASO.

\section{Supplementary information}

Supplementary information accompanies this paper at https://doi.org/10. 1186/s13643-020-01487-3.

Additional file 1. Appendix 1 MEdline search strategy 2 October 2018

Additional file 2. Appendix 2 Characteristics of included studies.

Additional file 3. Appendix 3 Characteristics of excluded studies with reasons

Additional file 4. Appendix 4 Summary of findings table: Arterial switch operation for transposition of the great arteries.

Additional file 5. Appendix 5 Sensitivity analysis for the main and secondary outcomes

Additional file 6. Appendix 6 Preferred Reporting Items for Systematic reviews and Meta-Analyses extension for Scoping Reviews (PRISMA-SCR) Checklist.

\section{Abbreviations}

MEDLINE: Medical Literature Analysis and Retrieval System Online; EMBASE: Excerpta Medica dataBASE; CINAHL: Cumulative Index to Nursing and Allied Health Literature; LILACS: Literature in the Health Sciences in Latin America and the Caribbean; TGA: Transposition of the great arteries; D-TGA: Dextrotransposition of the great arteries; L-TGA: Levo-transposition of the great arteries; ASO: Arterial switch operation; GRADE: Grading of Recommendations Assessment, Development and Evaluation; PROSPERO: International prospective register of systematic reviews; GDT: Guideline Development Tool; PRISMA: Preferred Reporting Items for Systematic Reviews and MetaAnalyses; Cl: Confidence interval; BSID: Bayley Scales of Infant Development; MDI: Mental Development Index; PDI: Physical Development Index; SD: Standard deviation

\section{Authors' contributions}

$L M, L T$ and $R D$ conceived the research idea. FM and $A L$ did the study searches and data abstraction, while LM helped with the arbitration of discrepancies. FM made the first draft. All authors reviewed several versions of the manuscript. All authors read and approved the final manuscript.

\section{Funding}

The authors did not receive any funding for this project.

\section{Availability of data and materials}

Data sharing is not applicable to this article as no datasets were generated or analysed during the current study. A list of additional material is found in the appendix, and references made to them within the manuscript.

\section{Ethics approval and consent to participate}

Being a scoping review of published literature, we did not need any ethical approval nor patient consent in order to conduct this study.

\section{Consent for publication}

Not applicable.

\section{Competing interests}

None.

\section{Author details}

'Department of Health Research Methods, Evidence and Impact, McMaster University, Hamilton, ON, Canada. ${ }^{2}$ Department of Obstetrics and Gynecology, Faculty of Medicines and Biomedical Sciences, University of Yaoundé 1, Yaoundé, Cameroon. ${ }^{3}$ Faculty of Health Sciences, University of Bamenda, Bamenda, Cameroon. ${ }^{4}$ Biostatistics Unit/FSORC, St Joseph Healthcare-Hamilton, 50 Charlton Avenue East, 3rd Floor Martha Wing, Room H321, Hamilton, ON L8N 4A6, Canada. ${ }^{5}$ Centre for Development of Best Practices in Health, Yaoundé Central Hospital, Yaoundé, Cameroon. ${ }^{6}$ Departments of Pediatrics and Anesthesia, McMaster University, Hamilton, Ontario, Canada.

Received: 4 February 2019 Accepted: 17 September 2020 Published online: 07 October 2020

References

1. Marlyn S. Woo JRS. Pulmonary manifestations of cardiac diseases. In: Nelson L. Turcios, M.D., F.C.C.P., and Robert J. Fink MD, editor. Pulmonary manifestations of pediatric diseases. Turcios and Fink; 2009. p. 79-97.

2. Barry D. Kussman FXM. Congenital Cardiac anesthesia. In: Smith's Anesthesia for infants and children. 8th ed. Philadelphia, PA 19103-2899: Elsevier Mosby; 2011.

3. Canfield MA, Honein MA, Yuskiv N, Xing J, Mai CT, Collins JS, Devine O, Petrini J, Ramadhani TA, Hobbs CA, et al. National estimates and race/ ethnic-specific variation of selected birth defects in the United States. Birth Defects Res A Clin Mol Teratol. 1999-2001;76:747-56.

4. Animasahun BA, Madise-Wobo AD, Gbelee $\mathrm{HO}$, SIO. Children with transposition of the great arteries: Should they actually be born in Nigeria? World J Cardiol. 2017:9(3):277-82

5. Hornung TS, Calder L. Congenitally corrected transposition of the great arteries. Heart. 2010:96(14):1154-61.

6. CA W, Warnes CA. Transposition of the great arteries. Circulation. 2006 Dec; 114(24):2699-709.

7. Bleich SN, Vercammen KA, Zatz LY, Frelier JM, Ebbeling CB, Peeters A. Interventions to prevent global childhood overweight and obesity: a systematic review. Lancet Diabetes Endocrinol. 2017;6(4):332-46.

8. Kulik TJ. Physiology of congenital heart disease in the neonate. In: Richard A. Polin, Steven H. Abman DR and WEB, editor. Fetal and Neonatal physiology. 5th ed. Elsevier Inc.; 2017.

9. Dean B. Andropoulos EAG. Congenital heart disease. In: Fleisher LA, editor Anesthesia and uncommon Diseases. 2012. p. 75-136.

10. de Laval MR. Lessons from the arterial-switch operation. Lancet. 2001; 357:1814

11. Pretre R, Tamisier D, Bonhoeffer P, Mauriat P, Pouard P, Sidi D, et al. Results of the arterial switch operation in neonates with transposed great arteries. Lancet (London, England). 2001 Jun;357(9271):1826-30.

12. Horer J, Schreiber C, Cleuziou J, Vogt M, Prodan Z, Busch R, et al. Improvement in long-term survival after hospital discharge but not in freedom from reoperation after the change from atrial to arterial switch for transposition of the great arteries. J Thorac Cardiovasc Surg. 2009 Feb; 137(2):347-54

13. Khairy P, Clair M, Fernandes SM, Blume ED, Powell AJ, Newburger JW, et al. Cardiovascular outcomes after the arterial switch operation for Dtransposition of the great arteries. Circulation. 2013 Jan;127(3):331-9.

14. Raissadati $\mathrm{A}$, Nieminen $\mathrm{H}$, Sairanen $\mathrm{H}$, Jokinen $\mathrm{E}$. Outcomes after the Mustard, Senning and arterial switch operation for treatment of transposition of the great arteries in Finland: a nationwide 4-decade perspective. Eur J Cardiothorac Surg. 2017 Sep;52(3):573-80.

15. Geva T, Nathan M. Elliott MJ. Vetter VL. the Current Era of the Arterial Switch Operation. 2015;64(5):498-511.

16. Mbuagbaw L, Forlemu-Kamwa D, Chu A, Thabane L, Dillenberg R. Outcomes after corrective surgery for congenital dextro-transposition of the great arteries using the arterial switch technique: A protocol for a scoping systematic review. BMJ Open. 2014;4(6):1-5.

17. Andrea C. Tricco; Erin Lillie; Wasifa Zarin; Kelly K. O'Brien; Heather Colquhoun; Danielle Levac; David Moher; Micah D.J. Peters; Tanya Horsley; Laura Weeks; Susanne Hempel et al. PRISMA Extension for Scoping Reviews (PRISMA-SCR): Checklist and Explanation. Ann Intern Med. 2018:169(7):467-73.

18. Lecompte $Y$, Zannini L, Hazan E, Jarreau MM, Bex JP, Tu TV, et al. Anatomic correction of transposition of the great arteries. J Thorac Cardiovasc Surg. 1981 Oct;82(4):629-31. 
19. Moons, P., Gewillig, M., Sluysmans, T., Verhaaren, H., Viart, P., Massin, M., Suys, B., Budts, W., Pasquet, A., De Wolf, D., Vliers A. Long term outcome up to 30 years after the Mustard or Senning operation: a nationwide multicentre study in Belgium. 2004;28:307-13.

20. Alsoufi B, Awan A, Al-Omrani A, Al-Ahmadi M, Canver CC, Bulbul Z, et al. The rastelli procedure for transposition of the great arteries: resection of the infundibular septum diminishes recurrent left ventricular outflow tract obstruction risk. Ann Thorac Surg. 2009 Jul:88(1):133-7.

21. Hazekamp MG, Nevvazhay T, Sojak V. Nikaidoh vs Reparation a l'Etage Ventriculaire vs Rastelli. Semin Thorac Cardiovasc Surg Pediatr Card Surg Annu. 2018 Mar;21:58-63.

22. DistillerSR, Evidence Partners. Ottawa, Canada; 2018. Available from: https://www.evidencepartners.com/.

23. Ankit Rohatgi. WebPlotDigitizer- Web Based Plot Digitizer. 2010. Available from: http://arohatgi.info/WebPlotDigitizer.

24. Hoy D, Brooks P, Woolf A, Blyth F, March L, Bain C, et al. Assessing risk of bias in prevalence studies : modification of an existing tool and evidence of interrater agreement. J Clin Epidemiol. 2012;65(9):934-9.

25. StataCorp. Stata Statistical Software: Release 13. College Station, TX: StataCorp LP.; 2013.

26. Yi YG, Sung IY, Yuk JS. Comparison of Second and Third Editions of the Bayley Scales in Children With Suspected Developmental Delay. 2018;42(2): 313-20.

27. Julian P.T Higgins, Douglas G. Altman JA. S. Assessing risk of bias in included studies. In: Higgins JPT GS, editor. Cochrane Handbook for Systematic Reviews of Interventions Version 510 Updated. The Cochrane Collaboration 2011. March 2011:2011.

28. Nyaga VN, Arbyn M, Aerts M. Metaprop : a Stata command to perform meta-analysis of binomial data. Arch Public Heal. 2014;72(39):1-10.

29. Guyatt GH, Oxman AD, Vist GE, Kunz R, Falck-Ytter Y, Alonso-Coello P, et al. GRADE: An emerging consensus on rating quality of evidence and strength of recommendations. Chinese J Evidence-Based Med. 2009;9(1):8-11.

30. GRADEpro GDT: GRADEpro Guideline Development Tool [Software]. McMaster University, 2015 (developed by Evidence Prime, Inc.). Available from gradepro.org.

31. Lim H-G, Kim W-H, Lee JR, et al. Long-term results of the arterial switch operation for ventriculo-arterial discordance. Eur J Cardio-thoracic Surg. 2013 Feb;43(2):325-34.

32. Fricke TA, Loyer BR, Huang L, Griffiths S, Yaftian N, Dalziel KM, et al. Longterm quality of life in adult survivors after the arterial switch operation. Eur J Cardiothorac Surg. 2018 May.

33. Kalfa D, Kasmi L, Geronikola N, Calderon J, Lambert V, Belli E, et al. Cognitive outcomes and health-related quality of life in adults two decades after the arterial switch operation for transposition of the great arteries. J Thorac Cardiovasc Surg. 2017 Sep;154(3):1028-35.

34. Ruys, T.P,E., van der Bosch, A.E., Cuypers, J.A., Witsenburg, M., Helbing, W.A. et al. Long-term outcome and quality of life after arterial switch operation: A prospective study with a historical comparison. Congenit Heart Dis. 2013; 8(3):203-210.

35. Gorler H, Ono M, Thies A, Lunkewitz E, Westhoff-Bleck M, et al. Long-term morbidity and quality of life after surgical repair of transposition of the great arteries: Atrial versus arterial switch operation. Interact Cardiovasc Thorac Surg. 2011;12(4):569-74

36. De Koning WB, Van Osch-Gevers M, Harkel AD, Van Domburg RT, Spijkerboer AW, et al. Follow-up outcomes 10 years after arterial switch operation for transposition of the great arteries: Comparison of cardiological health status and health-related quality of life to those of the a normal reference population. Eur J Pediatr. 2008;167(9):995-1004.

37. Hovels-Gurich HH, Konrad K, Wiesner M, Minkenberg R, Herpertz-Dahlmann B, BJ M, et al. Long term behavioural outcome after neonatal arterial switch operation for transposition of the great arteries. Arch Dis Child. 2002 Dec; 87(6):506-10.

38. Dunbar-Masterson C, Wypij D, Bellinger DC, Rappaport LA, Baker AL, Jonas RA, et al. General health status of children with D-transposition of the great arteries after the arterial switch operation. Circulation. 2001 Sep;104(12 Suppl 1):1138-42.

39. Massin, M.M. Midterm results of the neonatal arterial switch operation. A review. J Cardiovasc Surg (Torino). 1999;40(4):517-22.

40. Raja SG, Shauq A, Kaarne M. Outcomes after arterial switch operation for simple transposition. Asian Cardiovasc Thorac Ann. 2005 Jun;13(2):190-8.
41. van Wijk SWH. van der Stelt., ter Heide, H., Schoof, P.H., Doevendans, P.A., Meijboom, F.J. Sudden Death Due to Coronary Artery Lesions Long-term After the Arterial Switch Operation: A Systematic Review. Can J Cardiol. 2017:33(9):1180-7.

42. Moe TG. Bardo DME. Prog Cardiovasc Dis: Long-Term Outcomes of the Arterial Switch Operation for D-Transposition of the Great Arteries: 2018

43. Blume ED, Wernovsky G. Long-term results of arterial switch repair of transposition of the great vessels. Semin Thorac Cardiovasc Surg Pediatr Card Surg Annu. 1998;1:129-38.

44. Vargo P, Mavroudis C, Stewart RD, Backer CL. Late complications following the arterial switch operation. World J Pediatr Congenit Heart Surg. 2011 Jan 2(1):37-42.

45. Legendre A, Losay J, Touchot-Kone A, Serraf A, Belli E, Piot JD, et al. Coronary events after arterial switch operation for transposition of the great arteries. Circulation. 2003 Sep;108(Suppl):|l186-90.

46. Kasmi L, Calderon J, Montreuil M, Geronikola N, Lambert V, Belli E, et al. Neurocognitive and Psychological Outcomes in Adults With DextroTransposition of the Great Arteries Corrected by the Arterial Switch Operation. Ann Thorac Surg. 2018 Mar;105(3):830-6.

47. Daebritz SH, Nollert G, Sachweh JS, Engelhardt W, von Bernuth G, Messmer BJ. Anatomical risk factors for mortality and cardiac morbidity after arterial switch operation. Ann Thorac Surg. 2000 Jun;69(6):1880-6.

48. Popov AF, Tirilomis T, Giesler M, Oguz Coskun K, Hinz J, Hanekop GG, et al. Midterm results after arterial switch operation for transposition of the great arteries: a single centre experience. J Cardiothorac Surg. 2012 Sep;7:83.

49. Fteropoulli T, Stygall J, Cullen S, Deanfield J. Newman SP. Quality of life of adult congenital heart disease patients: a systematic review of the literature. 2013:473-85.

\section{Publisher's Note}

Springer Nature remains neutral with regard to jurisdictional claims in published maps and institutional affiliations.

Ready to submit your research? Choose BMC and benefit from:

- fast, convenient online submission

- thorough peer review by experienced researchers in your field

- rapid publication on acceptance

- support for research data, including large and complex data types

- gold Open Access which fosters wider collaboration and increased citations

- maximum visibility for your research: over $100 \mathrm{M}$ website views per year

At $\mathrm{BMC}$, research is always in progress.

Learn more biomedcentral.com/submissions 